\title{
Genetic mapping of ovary colour and quantitative trait loci for carotenoid content in the fruit of Cucurbita maxima Duchesne
}

\author{
Karolina Kaźmińska • Ewelina Hallmann • Anna Rusaczonek • \\ Aleksandra Korzeniewska • Mirosław Sobczak • Joanna Filipczak • \\ Karol Seweryn Kuczerski • Jarosław Steciuk • Monika Sitarek-Andrzejczyk • \\ Marek Gajewski • Katarzyna Niemirowicz-Szczytt • Grzegorz Bartoszewski $(\mathbb{C}$
}

Received: 6 April 2018 / Accepted: 13 August 2018/Published online: 27 August 2018

(C) The Author(s) 2018

\begin{abstract}
The high content of carotenoids, sugars, dry matter, vitamins and minerals makes the fruit of winter squash (Cucurbita maxima Duchesne) a valuable freshmarket vegetable and an interesting material for the food industry. Due to their nutritional value, long shelf-life and health protective properties, winter squash fruits have gained increased interest from researchers in recent years. Despite these advantages, the genetic and genomic resources available for C. maxima are still limited. The aim of this study was to use the genetic mapping approach to map the ovary colour locus and to identify the quantitative trait loci (QTLs) for high carotenoid content and flesh colour. An $\mathrm{F}_{6}$ recombinant inbred line (RIL) mapping
\end{abstract}

Electronic supplementary material The online version of this article (https://doi.org/10.1007/s11032-018-0869-z) contains supplementary material, which is available to authorized users.

K. Kaźmińska · A. Rusaczonek · A. Korzeniewska •

J. Filipczak · K. S. Kuczerski · J. Steciuk •

K. Niemirowicz-Szczytt • G. Bartoszewski $(\bowtie)$

Department of Plant Genetics, Breeding and Biotechnology,

Faculty of Horticulture Biotechnology and Landscape Architecture,

Warsaw University of Life Sciences, Warsaw, Poland

e-mail: grzegorz_bartoszewski@sggw.pl

E. Hallmann

Organic Food Division, Faculty of Human Nutrition and Consumer

Sciences, Warsaw University of Life Sciences, Warsaw, Poland

M. Sobczak

Department of Botany, Faculty of Agriculture and Biology, Warsaw University of Life Sciences, Warsaw, Poland population was developed and used for evaluations of ovary colour, carotenoid content and fruit flesh colour. SSR markers and DArTseq genotyping-by-sequencing were used to construct an advanced genetic map that consisted of 1824 molecular markers distributed across linkage groups corresponding to 20 chromosomes of C. maxima. Total map length was $2208 \mathrm{cM}$ and the average distance between markers was $1.21 \mathrm{cM}$. The locus affecting ovary colour was mapped at the end of chromosome 14. The identified QTLs for carotenoid content in the fruit and fruit flesh colour shared locations on chromosomes 2, 4 and 14. QTLs on chromosomes 2 and 4 were the most meaningful. A correlation was clearly

M. Sitarek-Andrzejczyk • M. Gajewski

Department of Vegetable and Medicinal Plants, Faculty of Horticulture Biotechnology and Landscape Architecture, Warsaw University of Life Sciences, Warsaw, Poland

Present Address:

K. S. Kuczerski

Department of Plant Physiology, Warsaw University of Life

Sciences, Warsaw, Poland

Present Address:

J. Steciuk

Institute of Biochemistry and Biophysics Polish Academy of Sciences, Warsaw, Poland 
confirmed between fruit flesh colour as described by the chroma value and carotenoid content in the fruit. A highdensity genetic map of $C$. maxima with mapped loci for important fruit quality traits is a valuable resource for winter squash improvement programmes.

Keywords Carotenoids · Cucurbita maxima .

Fruit colour - Genetic map · Molecular markers · Ovary colour $\cdot$ QTL

\section{Introduction}

Carotenoids comprise a large class of pigments that demonstrate great variability. They consist of eight isoprene units and are derived from the $\mathrm{C}_{40} \mathrm{H}_{56}$ basic structure that absorbs light from a part of the visible region of the electromagnetic spectrum $(400-500 \mathrm{~nm})$ (Britton 1995). Carotenoids are classified as carotenes and xanthophylls, where xanthophylls contain an oxygen-containing functional group (Bhosale and Bernstein 2005). Plant carotenoids are yellow, orange and red lipid-soluble pigments found in the chloroplasts and chromoplasts. Carotenoids, mainly xanthophylls, are parts of light-harvesting complexes and protect the photosynthetic apparatus against photo-oxidative damage (Britton 2008). Furthermore, carotenoids give colour to flowers and fruits; thus, they help attract pollinators and secure pollen grain transfer $(\mathrm{Lu}$ and $\mathrm{Li}$ 2008). These compounds also provide many important health benefits, e.g. carotenoids with provitamin A activity, mainly $\beta$-carotene, are essential components of the human diet (Bowen et al. 2015). The xanthophylls zeaxanthin and lutein, which are the pigmented components of the macula, provide protection against age-related macular degeneration (Abdel-Aal et al. 2013). These compounds are also precursors of apocarotenoids, which are involved in a wide range of biological processes, including plant development and growth, regulation of stress responses and contribution to the flavour and aroma of fruit and flowers (Gong et al. 2012; Lu and Li 2008; Tuteja 2007). The plant carotenoid biosynthetic pathway is localised in plastids, and the genes involved in this pathway are well characterised (Hirschberg 2001). The regulation of carotenoid biosynthesis has been widely investigated in many plant species, including in the tomato as a model system (Nisar et al. 2015; Yuan et al. 2015).
Cucurbita maxima Duchesne (winter squash, pumpkin, gourd) is an economically important crop species of the genus Cucurbita. Pumpkin and winter squash fruits are a valuable source of carotenoids, sugars, minerals and vitamins; thus, they are used as a fresh product and in the food processing industry. Pumpkin and winter squash are amongst the most frequently grown organic vegetables (Kopta et al. 2018). The nutritional value and flesh colour of $C$. maxima fruit are its most important quality traits with respect to consumer preference and acceptance (Nakkanong et al. 2012). Fruit flesh colour is positively correlated with carotenoid content, and a particular hue of the flesh can be related to the proportion of individual carotenoids (Paris 1994; Seroczyńska et al. 2006). The predominant carotenoids present in winter squash fruit are $\beta$-carotene, lutein and $\alpha$-carotene, although these carotenoids' compositions vary and depend on several factors, including the cultivar, growing conditions, harvest time, storage period and conditions (Biesiada et al. 2009; Bonina-Noseworthy et al. 2016; Murkovic et al. 2002; Nakkanong et al. 2012; Kreck et al. 2006). For example, in commercially grown cultivars, the content of $\beta$-carotene, which is a precursor of vitamin A, ranges from 1.4 to $8.4 \mathrm{mg}$ per $100 \mathrm{~g}$, with up to $12 \mathrm{mg}$ per $100 \mathrm{~g}$ in high $\beta$-carotene varieties such as the Polish cultivar 'Amazonka' (Murkovic et al. 2002; Sztangret et al. 2004).

Despite the economic importance of C. maxima, knowledge regarding its genomics is still far from complete, and only single genome sequence and few genetic maps are available (Ge et al. 2015; Singh et al. 2011; Sun et al. 2017; Zhang et al. 2015). The first high-density genetic map of $C$. maxima was developed for the $\mathrm{F}_{2}$ population derived from the cross of inbred line 'Rimu' and bush-type line SQ026. The application of genotyping-by-sequencing for this population resulted in a high-density genetic map and in identification of quantitative trait loci (QTLs) for dwarfism (Zhang et al. 2015). This and the second advanced $C$. maxima genetic maps were used to anchor scaffolds of the C. maxima 'Rimu' genome, which was recently sequenced (386.8 Mb) (Sun et al. 2017). Comparative analysis of the C. maxima and C. moschata genomes confirmed the allotetraploid nature of the Cucurbita genus (Sun et al. 2017). The allotetraploidy of $C$. maxima affects the genetic basis of complex traits such as carotenoid content in the fruit. A study of carotenogenesis genes in C. maxima fruit showed that homoeologous genes existed for this pathway, e.g. duplicated copies of the gene coding for 
phytoene synthase (PSY), which is a carotenoid biosynthesis-limiting enzyme, and only one of the homoeologs highly expressed in C. maxima fruit was identified (Sun et al. 2017).

QTL mapping is a commonly used approach to identify genetic regions responsible for important phenotypic variation. A common strategy of QTL mapping is the use of recombinant inbred lines (RILs) that allow for multiple self-pollination processes and thus can increase the number of recombination events, which result in finer mapping of QTLs and in the detection of possible QTL interactions. Moreover, RILs can be used repeatedly to investigate the QTLs of various phenotypes under different environments (Takuno et al. 2012).

In this study, a C. maxima advanced mapping population consisting of $\mathrm{F}_{6}$ RILs was developed and used for SSR and DArTseq genotyping to construct a highdensity genetic map in order to map the ovary colour locus and QTLs for carotenoid content and fruit flesh colour. This genetic map is a valuable genetic resource for C. maxima that can facilitate fruit-trait-orientated breeding programmes. The identified loci are so far the first fruit-related traits to be mapped in C. maxima.

\section{Materials and methods}

\section{Plant material}

An $\mathrm{F}_{6}$ population consisting of 92 RILs was developed from a cross between highly inbred lines 802 and 801 (both $>\mathrm{S}_{12}$ ). Line 802 was derived from the Hokkaido-type Japanese cultivar 'Uchiki Kuri' which is characterised by a yellow ovary colour, small fruit and orange fruit flesh. Line 801 was derived from an Eastern European landrace originating from the former Soviet Union with a light green ovary colour, large fruit and pale orange fruit flesh (Fig. 1a-f). A single $F_{1}$ plant produced from the $802 \times 801$ cross was selfpollinated to generate the $F_{2}$ population. $F_{2}$ individuals were self-pollinated up to the $\mathrm{F}_{6}$ generation by single seed descent. The parental lines were characterised by contrasting phenotypes for fruit traits and were genetically distant (Kaźmińska et al. 2017). All lines were grown and evaluated at the Wolica Experiment Station of the Department of Plant Genetics, Breeding and Biotechnology (DPGBB), Warsaw University of Life Sciences, Poland.
Phenotyping of the RIL population

A phenotypic evaluation of the parental lines and $F_{6}$ RILs was conducted during two field experiments using the randomised block method with three replicates (Exp. I performed in 2013 and Exp. II in 2014). In both experiments, the seeds were sown directly into the soil on 15 May at a spacing of $1.2 \mathrm{~m} \times 1.6 \mathrm{~m}$, with six plants per plot in each replica. The colour of the ovaries was evaluated visually during the flowering stage for at least three female flowers per plant (Fig. 1a, b). The fruit was harvested at the beginning of October, 70-80 days post anthesis, and stored for 6 weeks until 15 November in a plastic tunnel.

For the carotenoid measurements, fruit tissue was sampled after storage. For each line, six uniform fruits per each replica were chosen. Samples were taken from the sunny side of each fruit. After removing the skin and seeds, the fruit samples were shredded, pooled into three 5 -g samples and stored at $-80^{\circ} \mathrm{C}$ until use. The samples were homogenised in liquid nitrogen and $100 \mathrm{mg}$ of the homogenate was used for carotenoid extraction and HPLC analysis with the use of the Shimadzu Liquid Chromatography System (Shimadzu, Japan). The pigments were separated on a Synergi 4u MAX-RP 80A $250 \times 4.6$ column (Phenomenex, USA) at $30^{\circ} \mathrm{C}$. Solvent A (acetonitrile: methanol, 9:1 v/v) was used for $10 \mathrm{~min}$ to elute all of the xanthophylls, which was followed by solvent B (methanol: ethyl acetate, 68:32 $v / v$ ) for $10 \mathrm{~min}$ at a flow rate of $1 \mathrm{ml} \mathrm{min}^{-1}$ to extract the carotenoids. Absorbance spectra were recorded at $445 \mathrm{~nm}$ for xanthophyll and $450 \mathrm{~nm}$ for $\beta$-carotene by a diode array detector (Shimadzu, Japan). The carotenoid content was estimated for $\alpha$-carotene, $\beta$-carotene, lutein, violaxanthin, zeaxanthin and antheraxanthin based on carefully distinguishable peaks as the peak area per microgram of fresh weight (Rusaczonek et al. 2015) (Supplementary Fig. S1).

The fruit flesh colour was measured as described by Seroczyńska et al. (2006) with a HunterLab Miniscan XE spectrophotometer (HunterLab, USA) by using the International Commission on Illumination (CIE) system, where the value $\mathrm{L}^{*}$ describes lightness $\left(\mathrm{L}^{*}=0\right.$ for black, $L^{*}=100$ for white), $a^{*}$ describes colour intensity in red $\left(\mathrm{a}^{*}>0\right)$ or in green $\left(\mathrm{a}^{*}<0\right)$ and $\mathrm{b}^{*}$ describes colour intensity in yellow $\left(b^{*}>0\right)$ or in blue $\left(b^{*}<0\right)$ (Hunter and Harold 1987). The measurements were taken from 20-mm diameter fragments of the flesh surface. The instrument setup was Illuminant $=$ D65, 
Observer $=10^{\circ}$. Before taking the measurements, the device was standardised with a light trap and a white tile. Chroma values $\left(\mathrm{C}^{*}\right)$ were then calculated according to the formula $\mathrm{C}^{*}=\left(\mathrm{a}^{2}+\mathrm{b}^{*^{2}}\right)$. Universal HunterLab ${ }^{\mathrm{TM}}$ software was used to process the colour measurements.

Frequency distribution for all of the examined trait values and correlation analyses was obtained using Statistica 12 software (Statsoft Inc., USA). Correlations were calculated using the Pearson correlation coefficient at $p \leq 0.05$.

\section{DNA extraction}

Seeds were sown into a universal horticultural peatbased soil mixture (Karaska, Poland). Young leaves were collected from 3- to 4-week-old greenhousegrown plants, and at least four plants were used for each line. DNA was isolated using the GenElute Plant Genomic DNA Miniprep Kit (Sigma-Aldrich, USA) according to the manufacturer's instructions. DNA was quantified with a NanoDrop 2000 spectrophotometer (Thermo Fisher Scientific, USA) and diluted to a final concentration of $30 \mathrm{ng} \mathrm{Il}^{-1}$.

\section{SSR and DArTseq genotyping}

A total of 530 SSR markers obtained from previously published studies that included 500 genomic SSRs developed for Cucurbita moschata and Cucurbita pepo by Gong et al. (2008) and 30 EST-SSRs developed for C. pepo by Blanca et al. (2011) were tested on the parental lines to select polymorphic markers. Of these, a set of 36 markers was selected and used for genotyping and genetic map construction. Primers were commercially synthesised at Genomed S.A. (Warsaw, Poland) and Oligo.pl (Warsaw, Poland). PCR reactions were performed in a total volume of $15 \mu$ l according to DreamTaq polymerase (Thermo Fisher Scientific, USA) manufacturer's instructions as described before by Kaźmińska et al. (2017). The SSR PCR reactions were carried out using a Mastercycler EP Gradient S (Eppendorf, Germany) and the cycling programme as described by Pillen et al. (2000). The PCR amplicons were analysed using $6 \%$ polyacrylamide gel electrophoresis in $1 \times$ TBE buffer. The gels were stained using silver-staining method as described by Benbouza et al. (2006).

DArTseq genotyping-by-sequencing was conducted using a HiSeq2500 sequencing system (Illumina Inc.,
USA) atDiversity Arrays Technology Pty Ltd. (Canberra, Australia). In the DArTseq method, the genomecomplexity reduction step is applied in order to direct the analysis to hypomethylated, gene-rich genome regions (Wenzl et al. 2004). The DArTseq analytical pipeline was used to process the sequence reads and to identify polymorphisms, delivering SNPs and the presence or absence variation markers (silico-DArTs). SilicoDArTs were scored in a binary fashion, representing genetically 'dominant' markers, with ' 1 ' as the presence and ' 0 ' as the absence of a restriction fragment, whiles SNPs were coded as homozygotes similar to one parent as ' 0 ', to the other parent as ' 1 ', or heterozygotes as ' 2 '. Details of all the markers are provided in Supplementary Table S1.

Linkage map construction and QTL identification

A genetic linkage map was constructed with a minimum logarithm of odds (LOD) threshold of 6.0 and a recombination frequency value less than 0.4 using JoinMap 4 (Van Ooijen, 2006). The Kosambi mapping function was used for map construction (Kosambi 1944). The raw scores were inspected for any coding error and segregation distortion before being used as an input for the linkage analysis. All polymorphic markers that were heterozygous in any of the parents, markers with the same or missing alleles for the parents and duplicated markers were discarded. To detect segregation distortion, chi-square $\left(\chi^{2}\right)$ tests were computed for each SSR, SNP and silicoDArT. Highly distorted and unlinked markers were excluded from the analysis. MapChart 2.3 was used to visualise a constructed map for each linkage group (Voorrips 2002).

QTLs were identified based on the interval mapping (IM) model using MapQTL 5.0 (Van Ooijen 2004). The LOD threshold was determined by permutation analysis on the basis of 1000 permutations per trait at a significance level of $p \leq 0.05$ and $p \leq 0.01$. QTLs exceeding the threshold value $(p \leq 0.05)$ were considered significant. The percentage of phenotypic variance explained by QTLs (PVE, $R^{2}$ ) was estimated at the highest probability peak.

\section{Bioinformatics analysis}

Marker sequences were aligned to the C. maxima 'Rimu' genome v1.1 at the Cucurbit Genomics Database (http:// http://cucurbitgenomics.org/, Sun et al. 2017). The same 
genome sequence was used to identify annotated genomic regions corresponding to QTL intervals.

Microscopic analysis

Pieces of fruit tissue of the mapping population parental lines were dissected, processed for microscopic examination, and examined under light and electron transmission microscopes as described by Piszczek et al. (2011).

\section{Results}

Carotenoid content and fruit colour evaluation

The parental lines of the RIL population were significantly different in terms of carotenoid content in the fruit. Maternal line 802 accumulated significantly more carotenoids, predominantly $\beta$-carotene, lutein and $\alpha$ carotene as well as violaxanthin, zeaxanthin and antheraxanthin as compared to paternal line 801 . The fruit flesh colour described as the 'chroma' value was clearly different for these two lines, which was consistent with the visual assessment, i.e. 802 had intense orange fruit flesh whereas the flesh of 801 was pale orange (Fig. 1e, f). The RILs demonstrated variability of the examined traits and exceeded values represented by the parental lines for both years (Supplementary Fig. S2 and Table S2). Correlation analysis indicated seven traits that were highly correlated. The highest correlation coefficient, 0.73 , was found for $\beta$-carotene and for the fruit flesh colour chroma value (Table 1).

Genetic map construction

The genetic linkage map consisted of 36 SSRs, 1094 SNPs and 694 silicoDArTs - in total 1824 molecular markers distributed across 20 linkage groups (LGs) that were assigned to C. maxima chromosomes (Table 2, Supplementary Table S3). Individual LGs consisted of 51 to 109 markers for LG3 and LG15, respectively, with the mean of 91 markers per LG. The size of the LGs ranged from 70.6 to $222.9 \mathrm{cM}$ for LG15 and LG4, respectively. The total genetic length of the map was ca. $2208.3 \mathrm{cM}$. The average distance between the markers was $1.21 \mathrm{cM}$, and maximum spacing between the markers ranged from 3.9 to $14.77 \mathrm{cM}$. Chromosome 15 was the most saturated with 109 markers, with average marker spacing of $0.65 \mathrm{cM}$. The lowest average marker spacing of $2.1 \mathrm{cM}$ was for chromosome 4 . Marker positions were aligned with physical positions on the $C$. maxima 'Rimu' genome. Only chromosomes 15 and 17 contained regions with marker positions that were not fully compatible with the chromosomal positions (Supplementary Table S3). Based on chromosome size and LG length, the average recombination ratio was estimated at ca. $96 \mathrm{~kb}$ per $1 \mathrm{cM}$ (Table 2).

Mapping of ovary colour

Ovary colour was different for the parental lines of the mapping population - it was yellow for line 802 and light green for line 801 (Fig. 1a, b). The ovaries of the $F_{1}$ plants were yellow. Amongst the 92 evaluated $\mathrm{F}_{6}$ RILs, 45 lines possessed yellow ovaries and 47 had green ovaries (green and light green), which corresponded to a $1: 1$ segregation ratio $\left(\chi^{2}=0.043, p=0.05\right)$ (Supplementary Table S2). Based on the RIL evaluation, the locus affecting ovary colour was mapped at the end of chromosome 14 at $163.1 \mathrm{cM}$, with the closest marker 14-is20584145 linked at a distance of $4.8 \mathrm{cM}$ (Fig. 2, Supplementary Table 3).

Identification of QTLs for carotenoid content and fruit colour

Based on the phenotyping data for the RIL population, 13 QTLs related to $\beta$-carotene, lutein, $\alpha$-carotene, violaxanthin, zeaxanthin and antheraxanthin content in the fruit and 3 QTLs related to fruit flesh colour were identified (Fig. 2, Table 3 and Supplementary Fig. S3). The LOD values were similar for both years of the field experiments (Table 3, Supplementary Fig. S3).

The detected QTLs for $\beta$-carotene content, namely $q \beta$ car 2.1, q $\beta$ car4.1 and $q \beta$ car 14.1, had phenotypic variance explained values (PVE) ranging from 14.2 to $25.9 \%$. QTL q $\beta$ car 2.1 was less stable during the 2year experiments as compared to the other two. Similarly, three QTLs, namely qacar 2.1 , qacar4.1 and qacar14.1, for $\alpha$-carotene were detected in similar positions, and each explained from 14.9 to $30.1 \%$ of total phenotypic variation, with the less stable being

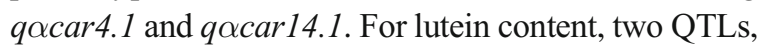
namely qlut2.1 and qlut4.1, with PVEs ranging from 17.1 to $28.4 \%$ were identified. Similarly, two QTLs for violaxanthin, i.e. qviol2.1 and qviol4.1, with PVEs from 21.4 to $27.3 \%$ as well as for antheraxanthin, i.e. qant 2.1 and qant4.1, with PVEs from 18.8 to $27.7 \%$ were 
Fig. 1 Morphological and microscopic characteristics of the RIL mapping population parental lines. a, b Ovaries of the mapping population paternal lines. Yellow ovary of maternal line 802 (a), light green ovary of paternal line 801 (b). Scalebars: $5 \mathrm{~cm}$. c-fFruits of C. maxima lines. Morphology (c and $\mathbf{d})$ and cross sections (e and $\mathbf{f}$ ) of mature fruits of the mapping population maternal line 802 (c and e) and paternal line 801 (d and f). Ruler size: $30 \mathrm{~cm} . \mathbf{g}$-j Fruit flesh anatomy and ultrastructure. Light ( $\mathbf{g}$ and $\mathbf{h}$ ) and transmission electron microscopy (i and $\mathbf{j}$ ) images showing the anatomy of fruit parenchyma (g and $\mathbf{h}$ ) and the ultrastructure of gerontoplasts (i and $\mathbf{j}$ ) in the fruit of maternal line 802 (g and i) and paternal line 801 (h and $\mathbf{j}$ ) of the RIL mapping population. The double tail arrows indicate plastids, the double head arrows mark thylakoids with osmiophilic content, the arrowheads point to plastoglobuli and arrows indicate thylakoids. $M$ mitochondrion, $\mathrm{Pl}$ plastid, $S$ starch. Scale bars: $100 \mu \mathrm{m}$ (g and h) and $1 \mu \mathrm{m}$ (i and $\mathbf{j})$
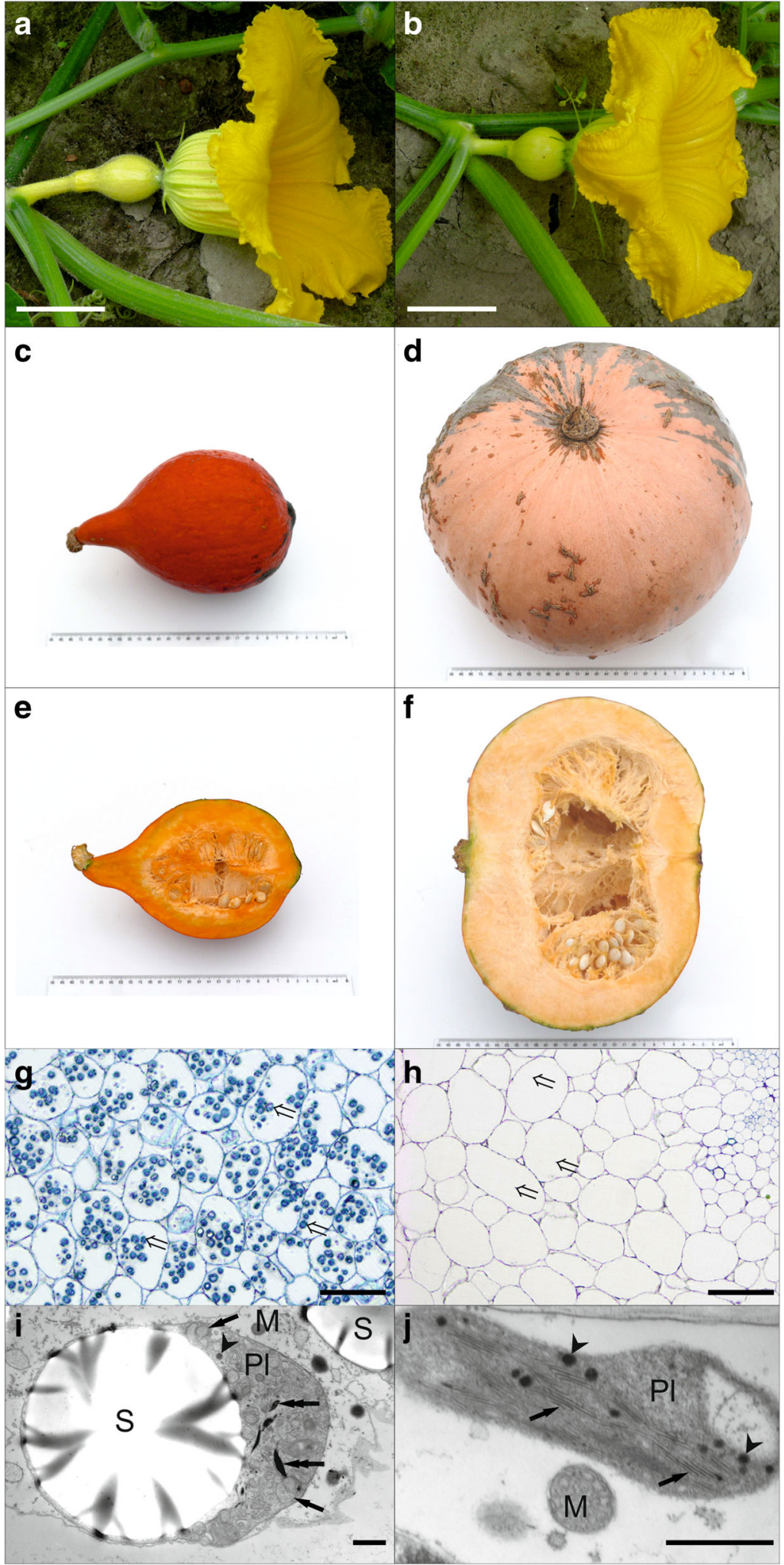
Table 1 Correlations for seven traits related to carotenoid content in C. maxima fruit. Correlation coefficient at $p \leq 0.05$

\begin{tabular}{|c|c|c|c|c|c|c|c|}
\hline Trait & $\beta$-Carotene & $\alpha$-Carotene & Lutein & Zeaxanthin & Antheraxanthin & Violaxanthin & Chroma \\
\hline$\beta$-Carotene & 1.00 & - & - & - & - & - & - \\
\hline$\alpha$-Carotene & 0.84 & 1.00 & - & - & - & - & - \\
\hline Lutein & 0.75 & 0.91 & 1.00 & - & - & - & - \\
\hline Zeaxanthin & 0.67 & 0.88 & 0.90 & 1.00 & - & - & - \\
\hline Antheraxanthin & 0.66 & 0.78 & 0.72 & 0.80 & 1.00 & - & - \\
\hline Violaxanthin & 0.78 & 0.82 & 0.82 & 0.79 & 0.92 & 1.00 & - \\
\hline Chroma & 0.73 & 0.70 & 0.63 & 0.67 & 0.66 & 0.70 & 1.00 \\
\hline
\end{tabular}

detected. For zeaxanthin, only a single QTL, qzea2.1, with PVEs from 18.9 to 28.2 was revealed. For fruit flesh colour, three QTLs, namely qch2.1, qch4.1 and qch14.1, which explained from 21.6 to $38.7 \%$ of total phenotypic variation, were identified.

All 16 identified QTLs for carotenoid content and fruit flesh colour corresponded to three chromosomal intervals: the first on chromosome 2 from 111.6 to
$142.1 \mathrm{cM}$, the second on chromosome 4 from 209.3 to the end, and the third on chromosome 14 from 144.8 to 152.9 cM (Fig. 2, Table 3, Supplementary Fig. 3). The sequences of markers flanking these three QTL intervals were aligned to the $C$. maxima 'Rimu' genome and were used to identify corresponding genomic regions. The first and largest interval corresponded to the $1.7 \mathrm{Mb}$ region of chromosome 2 and contained 346 genes.

Table 2 Basic characteristics of the C. maxima $802 \times 801$ genetic map. Linkage groups were anchored to C. maxima 'Rimu' chromosomes (Sun et al. 2017)

\begin{tabular}{|c|c|c|c|c|c|c|c|c|c|}
\hline \multirow{2}{*}{$\begin{array}{l}\text { Chromosome/ } \\
\text { linkage group }\end{array}$} & \multicolumn{4}{|c|}{ Marker number } & \multirow{2}{*}{$\begin{array}{l}\text { Total } \\
\text { distance }(\mathrm{cM})\end{array}$} & \multirow{2}{*}{$\begin{array}{l}\text { Average } \\
\text { spacing }(\mathrm{cM})\end{array}$} & \multirow{2}{*}{$\begin{array}{l}\text { Maximum } \\
\text { spacing }(\mathrm{cM})\end{array}$} & \multirow{2}{*}{$\begin{array}{l}\text { Length of } \\
\text { chromosome (bp) }\end{array}$} & \multirow[t]{2}{*}{$\mathrm{bp} / \mathrm{cM}$} \\
\hline & Total & SNPs & silicoDArTs & SSRs & & & & & \\
\hline 1 & 102 & 49 & 50 & 3 & 151.22 & 1.48 & 5.10 & $13,080,099$ & 86,498 \\
\hline 2 & 100 & 63 & 35 & 2 & 142.10 & 1.42 & 5.52 & $10,104,603$ & 71,107 \\
\hline 3 & 51 & 30 & 21 & 0 & 86.29 & 1.69 & 14.77 & $9,421,836$ & 109,180 \\
\hline 4 & 106 & 70 & 33 & 3 & 222.90 & 2.10 & 5.72 & $19,831,761$ & 88,970 \\
\hline 5 & 93 & 63 & 30 & 0 & 133.01 & 1.43 & 5.79 & $10,608,204$ & 79,755 \\
\hline 6 & 97 & 50 & 43 & 4 & 144.55 & 1.49 & 6.48 & $10,744,562$ & 74,332 \\
\hline 7 & 91 & 56 & 34 & 1 & 109.12 & 1.20 & 3.91 & $7,924,178$ & 72,618 \\
\hline 8 & 73 & 44 & 28 & 1 & 85.58 & 1.17 & 5.23 & $7,919,412$ & 92,538 \\
\hline 9 & 63 & 35 & 28 & 0 & 73.60 & 1.17 & 6.74 & $9,169,399$ & 124,584 \\
\hline 10 & 87 & 48 & 36 & 3 & 105.63 & 1.21 & 5.06 & $8,843,974$ & 83,727 \\
\hline 11 & 84 & 53 & 30 & 1 & 138.83 & 1.65 & 5.91 & $12,968,953$ & 93,417 \\
\hline 12 & 97 & 54 & 41 & 2 & 124.75 & 1.29 & 4.79 & $10,174,675$ & 81,560 \\
\hline 13 & 98 & 56 & 40 & 2 & 112.72 & 1.15 & 6.67 & $8,504,809$ & 75,452 \\
\hline 14 & 97 & 55 & 38 & 3 & 163.13 & 1.68 & 6.84 & $14,869,326$ & 91,148 \\
\hline 15 & 109 & 69 & 38 & 2 & 70.57 & 0.65 & 4.41 & $9,172,111$ & 129,964 \\
\hline 16 & 100 & 59 & 40 & 1 & 122.86 & 1.23 & 7.37 & $9,816,306$ & 79,900 \\
\hline 17 & 97 & 60 & 33 & 4 & 149.15 & 1.54 & 6.70 & $9,500,423$ & 63,698 \\
\hline 18 & 101 & 58 & 41 & 2 & 132.80 & 1.31 & 6.27 & $10,257,106$ & 77,236 \\
\hline 19 & 83 & 58 & 24 & 1 & 112.36 & 1.35 & 7.03 & $9,304,250$ & 82,805 \\
\hline 20 & 96 & 64 & 31 & 1 & 120.48 & 1.25 & 6.97 & $9,281,643$ & 77,038 \\
\hline Total & 1824 & 1094 & 694 & 36 & 2208.35 & & & $211,497,630$ & 95,772 \\
\hline
\end{tabular}


Chromosome 2

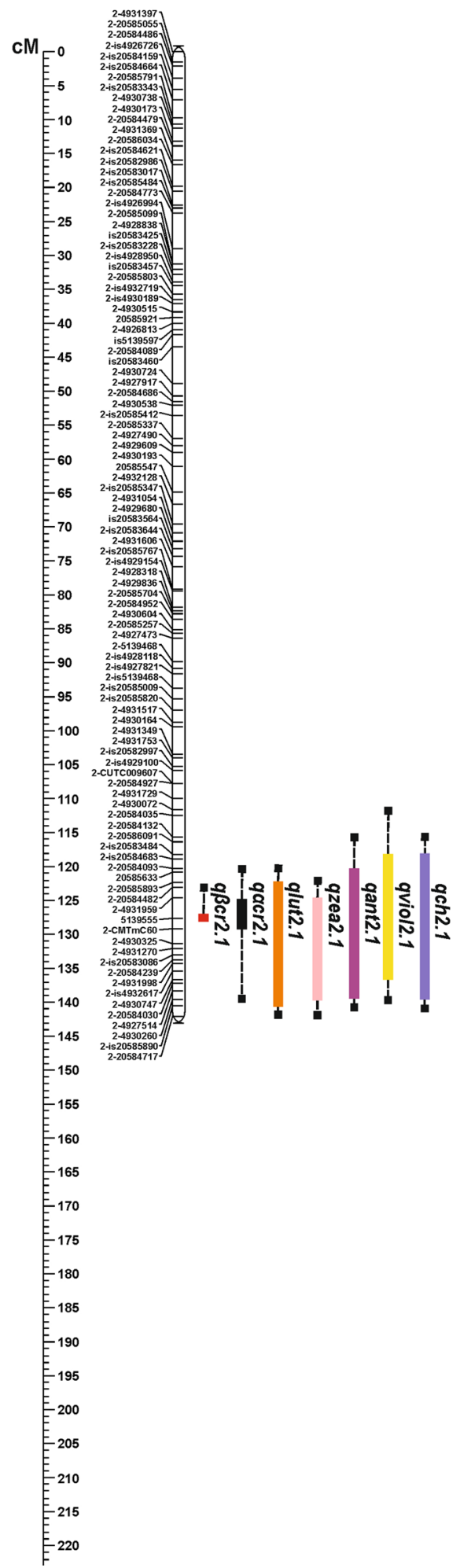

Chromosome 4

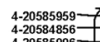

$4.20554596-$
$4-20585996-$

4.

4-20585302

4-is20583926 -

4-20584530-

4-20585889-

4-4931192-

4-is20583536

4-CMTP252-

4-is4928464

4-4931962-

4-20586169-

4-20585613-

$4-4927936-$

4-is4927971-

4-20585253 -

is 4 -is49258333

4-is 205845947

4-is 20583470

4-20585192-

4-20584517-

4 -is2058359

4-is20583965-

4-4930603-

4-20585639-7

4-205840

$4-4930896-$
is 20585173

4-is 4 -is $205583335895=$

4-20584988 -

$4-4931235$

$-4930148$

is 20583731

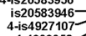

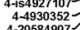

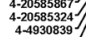

16-CMTM114
4932104
4

$4-4926903$
$4-492936$
4is4928372
is 20583083

is $4927197-$

4-20585555-

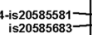

4-20583380-

$4-4929360-$

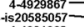

$4-43931992-2$
$4-20585954$

4.20538949
$4-2054149$
4

4-20584

$4-20584777$
$4-4926957$

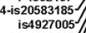

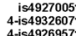

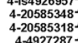

$4-4997287$
$4-4931362$

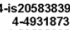

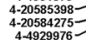

$4-5139552$

4-iss 20582998 -

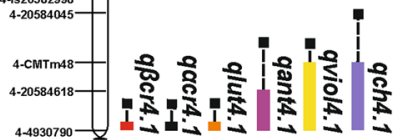

Chromosome 14

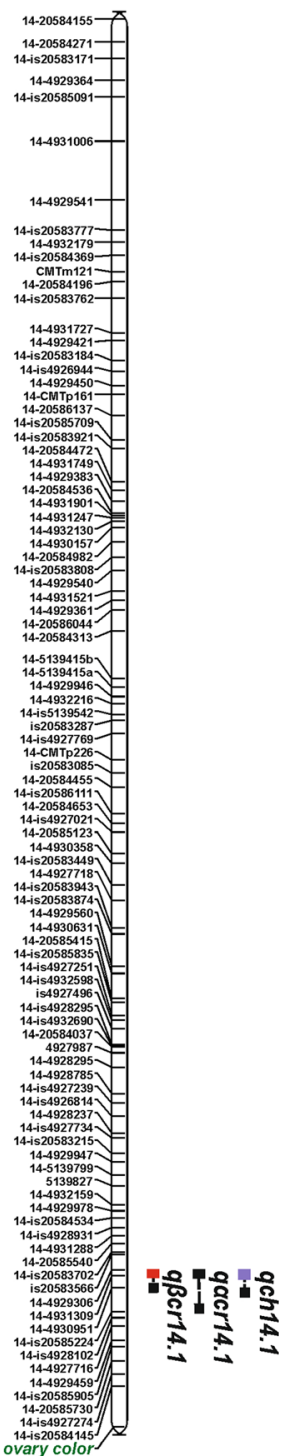

Fig. 2 Linkage distribution of mapped loci. Colour bars represent the location of identified QTLs for carotenoid content in the fruit and fruit colour measured as the chroma value (the largest intervals are marked) 


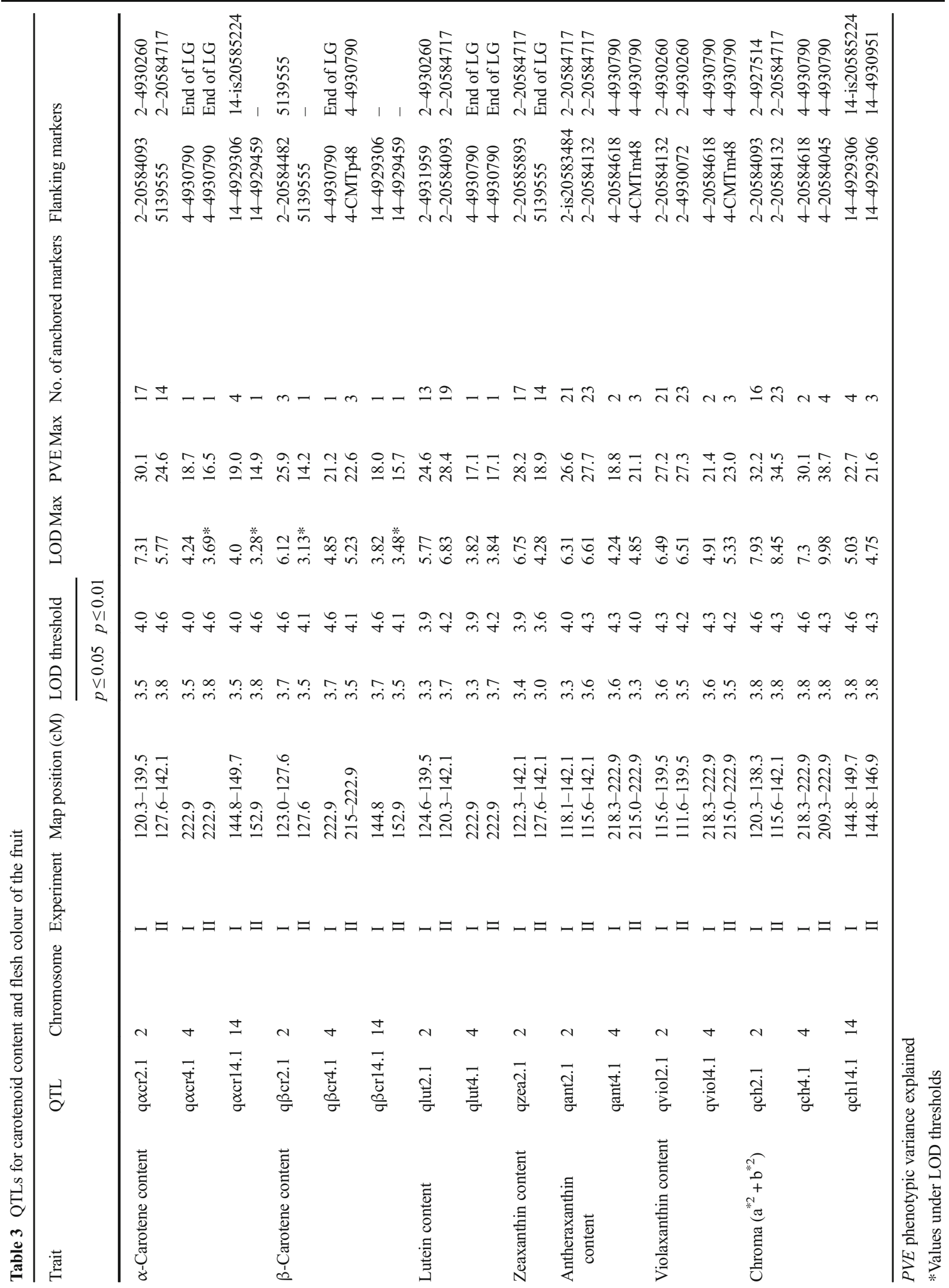


Two other QTL intervals corresponded to the $1 \mathrm{Mb}$ region on chromosome 4 and the $0.5 \mathrm{Mb}$ region on chromosome 14, including 163 and 102 genes, respectively (Supplementary Table S4). Carotenoid biosynthesis gene PSY (CmaCh04G022670) which codes for phytoene synthase as well as 12 transcriptional factors were found within the $1.0 \mathrm{Mb}$ genomic region on chromosome 4 . The genomic regions on chromosomes 2 and 14 contained 34 and 10 genes, respectively, encoding putative transcription factors (TFs) or proteins that can potentially regulate carotenoid biosynthesis and accumulation in C. maxima fruit.

\section{Structure of gerontoplasts}

Because the parental lines of the RIL mapping population significantly differed in amount and carotenoid composition, we conducted microscopic examinations of their fruit flesh (Fig. 1g-j). Light microscopy examinations showed that parenchymatic cells building the bulk of the fruit tissue did not differ in their cell dimensions and sizes of intercellular spaces (Fig. 1g, h). However, in the fruit cells of maternal line 802 , numerous large plastids with starch grains were present, whereas the fruit cells of paternal line 801 seemed to be almost 'empty'. Transmission electron microscopy showed that the cells of maternal line 802 fruit still contained a well-preserved protoplast with clearly recognisable organelles. The plastids contained large starch grains, but ultrastructurally, they resembled gerontoplasts (Fig. 1i). They were surrounded by a continuous envelope but their stroma were relatively electron-translucent, and the thylakoid system consisted of numerous vesicles and dilated thylakoid cisternae. Some of the cisternae were filled with osmiophilic carotenoids and the number of plastoglobuli was very low. In contrast, cells of paternal line 801 fruit contained almost no remnants of protoplasts and the only recognisable organelles were plastids, mitochondria and degraded nuclei (Fig. 1j). The plastids were very small and ultrastructurally resembled chloroplasts. Their envelope was still unbroken, the stroma was relatively electron-opaque and the thylakoids were arranged in parallel structures resembling grana. Electron-opaque plastoglobuli were small but present in high numbers.

\section{Discussion}

Despite the increasing economic importance of Cucurbita maxima in recent years, which is mostly related to $C$. maxima's high nutritional value, its good fruit storage properties and increasing share in the organic vegetable market, genetic studies for this cucurbit crop have been limited and so far only several genetic maps have been developed (Singh et al. 2011; Ge et al. 2015; Zhang et al. 2015; Sun et al. 2017). Recently, the genome of $C$. maxima line 'Rimu' was sequenced, thus becoming a valuable resource and tool for genetic and evolutionary studies on this species (Sun et al. 2017). Our study was focused on genetic mapping and identification of loci related to fruit traits in winter squash. An advanced RIL mapping population was developed and phenotyped. A set of SSR markers in combination with DArTseq genotyping-by-sequencing was used to construct a high-density genetic map and to map loci related to ovary colour, carotenoid content and fruit colour.

The genetic linkage map developed for the $F_{6}$ RIL mapping population complements previously reported C. maxima maps that were constructed for $\mathrm{F}_{2}$ populations (Singh et al. 2011; Ge et al. 2015; Zhang et al. 2015; Sun et al. 2017). The map was constructed for advanced $\mathrm{F}_{6}$ RILs developed from a cross of different origin inbred lines $\left(>S_{12}\right)$, i.e. the maternal line derived from the Japanese cultivar 'Uchiki Kuri' cultivated for fruit and the paternal line derived from an Eastern European local landrace also cultivated for fruit and characterised by contrasting ovary colour and fruit properties (Fig. 1a-f). The developed map is characterised by high marker density with a few gaps as compared to the other maps of C. maxima. The map consists of 1824 marker loci with $1.21 \mathrm{cM} /$ marker and $2208 \mathrm{cM}$ total length. The number of placed markers and density of the map is relatively high, although lower in comparison to the recently released and most advanced maps for other economically important Cucurbita species, namely C. pepo and C. moschata (Montero-Pau et al. 2017; Zhong et al. 2017).

In this study, a single locus affecting ovary colour in winter squash was mapped at the end of chromosome 14. This trait has not yet been investigated in C. maxima. Based on visual observation of the parental lines and the $\mathrm{F}_{1}$ hybrid, we noticed dominance of the yellow allele over the green allele, although ovaries with different shades of green, i.e. from light to dark green, were observed in the RILs, thus suggesting the existence of an additional locus modulating ovary colour in C. maxima. Moreover, adherence to a 
1:1 ratio in the RIL mapping population does not confirm dominance of the yellow allele, as complete genetic analysis is necessary in order to describe the gene related to ovary colour. Also, no correlation was found between ovary colour and fruit flesh colour in our study.

QTL mapping for six carotenoids ( $\alpha$-carotene, $\beta$ carotene, lutein, violaxanthin, zeaxanthin, and antheraxanthin) and for fruit flesh colour described as the chroma value revealed 16 QTLs that were all placed in three genomic regions located on chromosomes 2, 4 and 14. The QTL on chromosome 2 was most likely for the total carotenoid content since it was identified for all of the examined carotenes and xanthophylls. A similar situation was observed for the QTL region on chromosome 4, except there was no QTL for zeaxanthin, which could have been due to the low level of this compound in both parents. However, the QTL region on chromosome 14 was identified only for carotenes. QTL co-localisation could be explained by the fact that the values of all the traits were positively correlated (Table 1). QTL co-localisation for lutein, $\beta$-carotene and total carotenoid content was shown in C. moschata by Zhong et al. (2017). The QTLs that contributed to accumulation of all carotenoids in the fruit, explaining on average $25 \%$ of phenotypic variation for each measured carotenoid in each year except for $\beta$-carotene in 2015 were found on chromosome 2 (Table 3, Supplementary Fig. S3). QTLs found at the end of chromosome 4 also contributed to the accumulation of all carotenoids, except for zeaxanthin, explaining on average $20 \%$ of phenotypic variability for each investigated carotenoid (Table 3, Supplementary Fig. S3). The last QTLs were found on chromosome 14 and contributed only to the accumulation of $\alpha$ and $\beta$-carotene, explaining ca. $17 \%$ of phenotypic variability. QTLs located on chromosome 14 were less stable over the years (Table 3, Supplementary Fig. S3), and QTLs found on chromosomes 2 and 4 were the most significant and contributed to carotenoids accumulated in C. maxima fruit, explaining, together, ca. $45 \%$ of variation. The PVEs for individual QTLs related to major carotenoids, i.e. lutein, $\alpha$ and $\beta$-carotene accumulation, were similar to those reported for C. moschata (Zhong et al. 2017).

For the fruit flesh colour described as chroma values, three QTLs corresponding to carotenoid content were found, and the most significant QTLs were located, similarly, on chromosomes 2 and 4, explaining together $67 \%$ of phenotypic variation. A correlation between carotenoid content and fruit flesh colour measured as the chroma value was also found (Table 1). These results confirmed previous studies on C. maxima which showed a relationship between the chroma colour parameters of fruit flesh and carotenoid content (Seroczyńska et al. 2006). Thus, a fruit colour evaluation via the chroma value could be a useful method in breeding programmes in order to select winter squash accessions with a high carotenoid content. Recent QTL mapping studies on other Cucurbitaceae species, namely melon and watermelon, clearly supported the correlation between carotenoid accumulation and fruit flesh colour (Harel-Beja et al. 2010; Branham et al. 2017).

Carotenoid biosynthesis genes contribute to the accumulation of carotenoids in plants, although there are factors beyond this pathway that influence carotenoid accumulation. Amongst them, genes coding for proteins involved in transcriptional regulation at the biosynthesis level, carotenoid degradation, regulation of carotenoid sequestration and storage and plastid biogenesis may play crucial roles (Ellison et al. 2017). The mechanism of how carotenoids accumulate in $C$. maxima is even more complex because of this species' allotetraploid origin (Sun et al. 2017). In our study, the C. maxima 'Rimu' genome was used to identify regions corresponding to the detected QTLs and to mine these regions for genes potentially involved in carotenoid biosynthesis and accumulation.

The PSY gene that encodes phytoene synthase is a key gene in carotene biogenesis. It was found within the genomic region on chromosome 4, which corresponds to major QTLs for all of the investigated carotenoids, except for zeaxanthin. Phytoene synthase is a rate-limiting enzyme and therefore plays an important function in the formation of flowers and fruit colour (Nakkanong et al. 2012; Nisar et al. 2015; Yuan et al. 2015). Recently, Sun et al. (2017), based on genomic and transcriptomic analyses, described five $P S Y$ genes in C. maxima, but only one was highly expressed in the fruit. Thus, it is likely that the PSY gene located within the QTL on chromosome 4 is the key gene for carotenoid biosynthesis in C. maxima fruit. Interestingly, the pattern of transcription factors surrounding this gene within the QTL interval was very similar to that found in the confidence region of 
major QTL associated with $\beta$-carotene accumulation in watermelon (Branham et al. 2017).

One of the processes that can affect carotenoid accumulation is the regulation of plastid biogenesis. $T F$ genes were found within the QTL region on chromosome 2 encoding the Arabidopsis PseudoResponse Regulator (APRR). The genes were found in five copies next to one another in the QTL region with the highest LOD score. It was shown that APRR2-like TFs regulate plastid size, thus influencing the content of chlorophylls and carotenoids (Fukushima et al. 2009; Nadakuduti et al. 2014). Additionally, in this region, the STAY-GREEN $(S G R)$ gene encoding the magnesium dechelatase that regulates chlorophyll degradation and suppresses PSY expression during fruit ripening in tomato was found (Luo et al. 2013; Shimoda et al. 2016). Morever, within the QTL regions on chromosomes 2 and 14, genes encoding knotted-like homeobox $(K N O X)$, dystroglycan $(D A G)$ and the plastid DEAD box protein involved in fruit plastid development and differentiation were found (Chatterjee et al. 1996; Nadakuduti et al. 2014; Wang et al. 2000). Interestingly, in our study, a microscopic evaluation of the fruit sections for the parental lines showed large differences in the number, ultrastructure and size of the carotenoid-containing gerontoplasts, which suggests that the genes encoding TFs or proteins involved in plastid biogenesis, particularly those controlling the size and number of plastids, could be key factors responsible for carotenoid accumulation in C. maxima fruit (Fig. $1 \mathrm{~g}-\mathrm{j}$ ). Particularly intriguing is the cluster of $A P R R$ genes and $S G R$ gene found on chromosome 2. Moreover, in the QTL region on chromosome 2, we found genes involved in lightsignal transduction pathways, namely DET1 (De-Etiolated Homologue 1), DDB1 (UV-Damaged DNABinding Protein 1) and COP1 (E3 ubiquitin-protein ligase). DDB1 and DET1, which control plastid number and size, in addition to COP1 influence carotenoid content in tomato fruit (Davuluri et al. 2005; Wang et al. 2008; Ye et al. 2015).

A large number of genes coding other TFs or regulatory proteins that could affect carotenoid biosynthesis and accumulation were found within QTLs corresponding to genomic regions on chromosomes 2, 4 and 14. A group of $T F S$ that may interact in a different manner with structural genes involved in the carotenoid biosynthesis pathway was found, including the CCT domain containing TFS (CONSTANS, CO-like and Timing of CAB expression 1 (TOC1)), zinc finger proteins (CCCH-like, $\mathrm{C} 2 \mathrm{H} 2-$ like and SQUAMOSA-PROMOTER BINDING P R O T E IN ) , MYB $, B 3, b H L H, T C P$, DEHYDRATATION-INDUCED TF and MADS-box TFs (Ye et al. 2015; Ellison et al. 2017). Also, several $T F s$ that may affect carotenoid accumulation through regulation of fruit ripening were detected within the QTL intervals on chromosomes 2, 4 and 14. This group included ethylene response factors $(E t R F s)$ and the NAC transcription factor affecting ethylene synthesis and carotenoid accumulation (Lee et al. 2012; Ye et al. 2015; Zhu et al. 2014).

The RIL mapping population and genetic map as developed in this study can contribute to elucidating the genetic mechanisms controlling carotenoid accumulation in the fruit of the allotetraploid cucurbit C. maxima. The identified SNP and SSR markers linked to major QTLs located on chromosomes 2 and 4 could facilitate map saturation and fine mapping towards QTL dissection and identification of key genes controlling carotenoid biosynthesis and accumulation in C. maxima. Based on the C. maxima 'Rimu' genome annotation, we assumed that the PSY gene located on chromosome 4 as well as genes encoding transcriptional factors or proteins involved in plastid biogenesis possibly play an important role in carotenoid accumulation in winter squash fruit. These genes could be interesting candidates for associated mapping and genetic diversity studies within $C$. maxima.

Authors' contribution KK performed the research, analysed the data and participated in writing the manuscript. EH and AR conducted the carotenoid content analysis. AK guided RIL development and phenotyping. MS performed the microscopic analysis. JF, KSK and JS participated in SSR genotyping. MS-A and MG performed the fruit colour measurements. $\mathrm{KN}-\mathrm{S}$ participated in the experimental design. GB participated in designing and guiding the experiments, data analysis and in writing the manuscript. All of the authors read and approved the manuscript.

Funding This research study was supported partly by the Polish Ministry of Agriculture and Rural Development grant titled "Creating the scientific basis for biological progress and conservation of plant genetic resources as a source of innovation to support sustainable agriculture and food security for the country - protection of crop genetic resources". The study also benefited from networking activities within COST Action CA15136 (EUROCAROTEN). 


\section{Compliance with ethical standards}

Conflict of interest The authors declare that they have no conflicts of interest.

Ethical standards The experiments conducted in this study comply with domestic and international laws.

Open Access This article is distributed under the terms of the Creative Commons Attribution 4.0 International License (http:// creativecommons.org/licenses/by/4.0/), which permits unrestricted use, distribution, and reproduction in any medium, provided you give appropriate credit to the original author(s) and the source, provide a link to the Creative Commons license, and indicate if changes were made.

\section{References}

Abdel-Aal E-SM, Akhtar H, Zaheer K, Ali R (2013) Dietary sources of lutein and zeaxanthin carotenoids and their role in eye health. Nutrients 5:1169-1185

Benbouza H, Jacquemin J, Baudoin J, Mergeai G (2006) Optimization of reliable, fast, cheap and sensitive silver staining method to detect SSR markers in polyacrylamide gels. Biotechnol Agron Soc Environ 10:77-81

Bhosale P, Bernstein PS (2005) Microbial xanthophylls. Appl Microbiol Biotechnol 68:445-455

Biesiada A, Nawirska A, Kucharska A, Sokół-Łętowska A (2009) The effect of nitrogen fertilization methods on yield and chemical composition of pumpkin (Cucurbita maxima) fruits before and after storage. Veg Crops Res Bull 70:203-211

Blanca J, Cañizares J, Roig C, Ziarsolo P, Nuez F, Picó B (2011) Transcriptome characterization and high throughput SSRs and SNPs discovery in Cucurbita pepo (Cucurbitaceae). BMC Genomics 12:104

Bonina-Noseworthy J, Loy JB, Curran-Celentano J, Sideman R, Kopsell DA (2016) Carotenoid concentration and composition in winter squash: variability associated with different cultigens, harvest maturities, and storage times. HortScience 51:472-480

Bowen P, Stacewicz-Sapuntzakis M, Diwadkar-Navsariwala V (2015) Carotenoids in human nutrition. In: Chen C (ed) Pigments in fruits and vegetables. Springer, New York, pp $31-67$

Branham S, Vexler L, Meir A, Tzuri G, Frieman Z, Levi A, Wechter WP, Tadmor Y, Gur A (2017) Genetic mapping of a major codominant QTL associated with $\beta$-carotene accumulation in watermelon. Mol Breed 37:146

Britton G (1995) Structure and properties of carotenoids in relation to function. FASEB J 15:1551-1558

Britton G (2008) Functions of intact carotenoids. In: Britton G, Liaaen-Jensen S, Pfander H (eds) Carotenoids. Carotenoids vol 4. Birkhäuser, Basel, pp 189-212

Chatterjee M, Sparvoli S, Edmunds C, Garosi P, Findlay K, Martin C (1996) DAG, a gene required for chloroplast differentiation and palisade development in Antirrhinum majus. EMBO J 15:4194-4207
Davuluri GR, Tuinen A, Fraser PD, Manfredonia A, Newman R, Burgess D, Brummell DA, King SR, Palys J, Uhlig J, Bramley PM, Pennings HMJ, Bowler C (2005) Fruitspecific RNAi-mediated suppression of DET1 enhances carotenoid and flavonoid content in tomatoes. Nat Biotechnol 23:890-895

Ellison S, Senalik D, Bostan H, Iorizzo M, Simon P (2017) Fine mapping, transcriptome analysis, and marker development for $Y 2$, the gene that conditions $\beta$-carotene accumulation in carrot (Daucus carota L.). G3-Genes Genom Genet 7:26652675

Fukushima A, Kusano M, Nakamichi N, Kobayashi M, Hayashi N, Sakakibara H, Mizuno T, Saito K (2009) Impact of clockassociated Arabidopsis pseudoresponse regulators in metabolic coordination. Proc Natl Acad Sci U S A 106:72517256

Ge Y, Li X, Yang XX, Cui CS, Qu SP (2015) Genetic linkage map of Cucurbita maxima with molecular and morphological markers. Genet Mol Res 14:5480-5484

Gong L, Stift G, Kofler R, Pachner M, Lelley T (2008) Microsatellites for the genus Cucurbita and SSR-based genetic linkage map of Cucurbita pepo L. Theor Appl Genet 117:37-48

Gong L, Yang YJ, Zhou J (2012) Genes involved in the synthesis and signaling pathway of strigolactone, a shoot branching inhibitor. Biol Plant 56:210-214

Harel-Beja R, Tzuri G, Portnoy V, Lotan-Pompan M, Lev S, Cohen S, Dai N, Yeselson L, Meir A, Libhaber SE, Avisar E, Melame T, van Koert P, Verbakel H, Hofstede R, Volpin H, Oliver M, Fougedoire A, Stalh C, Fauve J, Copes B, Fei Z, Giovannoni J, Ori N, Lewinsohn E, Sherman A, Burger J, Tadmor Y, Schaffer AA, Katzir N (2010) A genetic map of melon highly enriched with fruit quality QTLs and EST markers, including sugar and carotenoid metabolism genes. Theor Appl Genet 121:511-533

Hirschberg J (2001) Carotenoid biosynthesis in flowering plants. Curr Opin Plant Biol 4:210-218

Hunter RS, Harold R (1987) The measurement of appearance, 2nd edn. Wiley, New York

Kaźmińska K, Sobieszek K, Targońska-Karasek M, Korzeniewska A, Niemirowicz-Szczytt K, Bartoszewski G (2017) Genetic diversity assessment of a winter squash and pumpkin (Cucurbita maxima Duchesne) germplasm collection based on genomic Cucurbita-conserved SSR markers. Sci Hortic 219:37-44

Kopta T, Hic P, Šlosár M, Pokluda R (2018) Quality changes in organic and conventional Hokkaido pumpkin (Cucurbita maxima Duch.) during storage. Biol Agric Hortic 34:1-9

Kosambi DD (1944) The estimation of map distance from recombination values. Ann Eugen 112:172-175

Kreck M, Kürbel P, Ludwig M, Paschold PJ, Dietrich H (2006) Identification and quantification of carotenoids in pumpkin species (Cucurbita maxima L.) and their juices by liquid chromatography with ultraviolet-diode array detection. J Appl Bot Food Qual 80:93-99

Lee JM, Joung JG, McQuinn R, Chung MY, Fei Z, Tieman D, Klee H, Giovannoni J (2012) Combined transcriptome, genetic diversity and metabolite profiling in tomato fruit reveals that the ethylene response factor SIERF6 plays an important role in ripening and carotenoid accumulation. Plant J 70:191204 
Lu S, Li L (2008) Carotenoid metabolism: biosynthesis, regulation, and beyond. J Integr Plant Biol 50:778-785

Luo Z, Zhang J, Li J, Yang C, Wang T, Ouyang B, Li H, Giovannoni J, Ye Z (2013) A STAY-GREEN protein SISGR1 regulates lycopene and $\beta$-carotene accumulation by interacting directly with SIPSY1 during ripening processes in tomato. New Phytol 198:442-452

Montero-Pau J, Blanca J, Esteras C, Martínez-Pérez EM, Gómez P, Monforte AJ, Cañizares J, Picó B (2017) An SNP-based saturated genetic map and QTL analysis of fruit-related traits in zucchini using genotyping-by-sequencing. BMC Genomics 18:94

Murkovic M, Mülleder U, Neunteufl H (2002) Carotenoid content in different varieties of pumpkins. J Food Compos Anal 15: 633-638

Nadakuduti SS, Holdsworth WL, Klein CL, Barry CS (2014) $K N O X$ genes influence a gradient of fruit chloroplast development through regulation of GOLDEN2-LIKE expression in tomato. Plant J 78:1022-1033

Nakkanong K, Yang JH, Zhang MF (2012) Carotenoid accumulation and carotenogenic gene expression during fruit development in novel interspecific inbred squash lines and their parents. J Agric Food Chem 60:5936-5944

Nisar N, Li L, Lu S, Khin NC, Pogson BJ (2015) Carotenoid metabolism in plants. Mol Plant 8:68-82

Paris HS (1994) Genetic analysis and breeding of pumpkins and squash for high carotene content. In: Linskens HF, Jackson JF (eds) Modern methods of plant analysis, Vegetables and vegetable products, vol 16. Springer Verlag, Berlin, pp 93115

Pillen K, Binder A, Kreuzkam B, Ramsay L, Waugh R, Förster J, Léon J (2000) Mapping new EMBL derived barley microsatellites and their use in differentiating German barley cultivars. Theor Appl Genet 101:652-660

Piszczek E, Dudkiewicz M, Sobczak M (2011) Molecular cloning and phylogenetic analysis of cereal type II metacaspase cDNA from wheat (Triticum aestivum). Biol Plant 55:614624

Rusaczonek A, Czarnocka W, Kacprzak S, Witoń D, Ślesak I, Szechyńska-Hebda M, Gawroński P, Karpiński S (2015) Role of phytochromes A and B in the regulation of cell death and acclimatory responses to UV stress in Arabidopsis thaliana. J Exp Bot 66:6679-6695

Seroczyńska A, Korzeniewska A, Sztangret-Wiśniewska J, Niemirowicz-Szczytt K, Gajewski M (2006) Relationship between carotenoids content and flower or fruit flesh colour of winter squash (Cucurbita maxima Duch.). Folia Hortic 18: $51-61$

Shimoda Y, Ito H, Tanaka A (2016) Arabidopsis Stay-Green, mendel's green cotyledon gene, encodes magnesiumdechelatase. Plant Cell 28:2147-2160

Singh AK, Singh R, Weeden NF, Robinson RW, Singh NK (2011) A linkage map for Cucurbita maxima based on randomly amplified polymorphic DNA (RAPD) markers. Indian J Hortic 68:44-50
Sun S, Wu S, Zhang G, Jiao C, Guo S, Ren Y, Zhang J, Zhang H, Gong G, Jia Z, Zhang F, Tian J, Lucas WJ, Doyle JJ, Li H, Fei Z, Xu T (2017) Karyotype stability and unbiased fractionation in the paleo-allotetraploid Cucurbita genomes. Mol Plant 10:1293-1306

Sztangret J, Korzeniewska A, Horbowicz M, Niemirowicz-Szczytt K (2004) Comparison of fruit yields and carotenoids content in new winter squash hybrids (Cucurbita maxima Duch.). Veg Crops Res Bull 61:51-60

Takuno S, Terauchi R, Innan H (2012) The power of QTL mapping with RILs. PLoS One 7:e46545

Tuteja N (2007) Abscisic acid and abiotic stress signaling. Plant Signal Behav 2:135-138

Van Ooijen JW (2004) MapQTL 5: software for the mapping of quantitative trait loci in experimental populations. Kyazma $\mathrm{BV}$, Wageningen

Van Ooijen JW (2006) JoinMap 4: software for the calculation of genetic linkage maps in experimental populations. Kyazma $\mathrm{BV}$, Wageningen

Voorrips RE (2002) MapChart: software for the graphical presentation of linkage maps and QTLs. J Hered 93:77-78

Wang Y, Duby G, Purnelle B, Boutry M (2000) Tobacco VDL gene encodes a plastid DEAD box RNA helicase and is involved in chloroplast differentiation and plant morphogenesis. Plant Cell 12:2129-2142

Wang S, Liu J, Feng J, Niu X, Giovannoni J, Liu Y (2008) Altered plastid levels and potential for improved fruit nutrient content by down regulation of the tomato DDB1-interacting protein CUL4. Plant J 55:89-103

Wenzl P, Carling J, Kudrna D, Jaccoud D, Huttner E, Kleinhofs A, Kilian A (2004) Diversity arrays technology (DArT) for whole-genome profiling of barley. Proc Natl Acad Sci U S A 101:9915-9920

Ye J, Hu T, Yang C, Li H, Yang M, Ijaz R, Ye Z, Zhang Y (2015) Transcriptome profiling of tomato fruit development reveals transcription factors associated with ascorbic acid, carotenoid and flavonoid biosynthesis. PLoS One 10:e0130885

Yuan H, Zhang J, Nageswaran D, Li L (2015) Carotenoid metabolism and regulation in horticultural crops. Hortic Res 2: 15036

Zhang G, Ren Y, Sun H, Guo S, Zhang F, Zhang J, Zhang H, Jia Z, Fei Z, Xu Y, Li H (2015) A high-density genetic map for anchoring genome sequences and identifying QTLs associated with dwarf vine in pumpkin (Cucurbita maxima Duch). BMC Genomics 16:1101

Zhong YJ, Zhou YY, Li JX, Yu T, Wu TQ, Luo JN, Luo SB, Huang HX (2017) A high-density linkage map and QTL mapping of fruit-related traits in pumpkin (Cucurbita moschata Duch). Sci Rep 7:12785

Zhu M, Chen G, Zhou S, Tu Y, Wang Y, Dong T, Hu Z (2014) A new tomato NAC (NAM/ATAF1/2/CUC2) transcription factor, SINAC4, functions as a positive regulator of fruit ripening and carotenoid accumulation. Plant Cell Physiol 1:119135 